\title{
Suspicious T1 colon cancer with synchronous liver metastasis not detected by preoperative imaging study
}

\author{
Chan Hee Park', So Hyang Moon', Hye Won Lee², Sung Uk Bae', Woon Kyung Jeong', Seong Kyu Baek' \\ Departments of 'Surgery and 'Pathology, Keimyung University Dongsan Medical Center, Keimyung University School of Medicine, Daegu, Korea
}

\begin{abstract}
Colorectal carcinoma invading the submucosa but not the muscularis propria (pT1) represents the earliest form of clinically relevant colorectal cancer in most patients. $\mathrm{T1}$ colorectal cancer with synchronous liver metastasis is considered to be rare. We report a rare case of T1 colon cancer with synchronous liver metastasis not detected by preoperative imaging study. A 54-year-old male patient presented to our department for treatment of sigmoid colon cancer following an endoscopic submucosal dissection. Histopathological examination revealed the pedunculated mass was moderately differentiated adenocarcinoma without lymphovascular invasion and the depth of submucosal invasion was 2,000 $\mu \mathrm{m}$, the resection margin was not involved. We performed a laparoscopic anterior resection with lymph node dissection. After the 3 months, the patient's carcinoembryonic antigen level elevated from 1.4 to $7.26 \mathrm{ng} / \mathrm{mL}$ (normal level: $<1.5 \mathrm{ng} / \mathrm{mL}$ ) and the abdominal computed tomography and FDG-PET/CT (positron emission tomography-computed tomography) showed multiple hepatic metastases in both hepatic lobes (SUVmax: 5.6) without evidence of local recurrence or lymphadenopathy. We strongly suspected a synchronous liver metastasis not detected by imaging study as opposed to a systemic recurrence. Therefore, evaluation and follow-up protocol of T1 colorectal cancer should be changed for discovery and prediction of synchronous liver metastasis; because we cannot exclude the possibility of synchronous liver metastasis.
\end{abstract}

Keywords: T1 colorectal cancer, Synchronous liver metastasis, Preoperative imaging study

\section{INTRODUCTION}

Colorectal cancer is one of the most common cancers worldwide, particularly in the economically developed world [1]. Recent developments in the diagnosis of colorectal cancer have led to an increase in the detection of early-stage cancer, especially pathologi-

Received: Jun 16, 2019 Revised: Oct 10, 2019 Accepted: Nov 14, 2019

Correspondence to: Seong Kyu Baek

Department of Surgery, Keimyung University Dongsan Medical Center, Keimyung University School of Medicine, 1095 Dalgubeol-daero, Dalseogu, Daegu 42601, Korea

Tel: +82-53-258-4709, Fax: +82-53-258-4708

E-mail:sgbeak@dsmc.or.kr

ORCID: Chan Hee Park (https://orcid.org/0000-0003-1125-2476), So Hyang Moon (https://orcid.org/0000-0001-6570-6176), Hye Won Lee (https://orcid.org/00000001-8540-524X), Sung Uk Bae (https://orcid.org/0000-0002-7876-4196), Woon Kyung Jeong (https://orcid.org/0000-0001-8421-218X), Seong Kyu Baek (https:// orcid.org/0000-0001-6427-8675)

Copyright (C) 2019 Korean Society of Surgical Oncology

This is an Open Access article distributed under the terms of the Creative Commons Attribution Non-Commercial License (http://creativecommons.org/licenses/by-nc/4.0) which permits unrestricted non-commercial use, distribution, and reproduction in any medium, provided the original work is properly cited. cally T1. Patients with T1 colorectal cancer have a good prognosis, and the 5 -year recurrence rate has been reported to be $1.3 \%$ after radical surgery [2].

The liver is the first and most common site of distant metastasis from colorectal cancer, involving up to $70 \%$ of patients and is the major cause of death [3-5]. The incidence of synchronous liver metastases in colorectal cancer has been reported to be about 30\% to $40 \%$ [4]. But, T1 colorectal cancer with synchronous liver metastasis is considered to be rare [6]. The form of metastasis from T1 colorectal cancer is mainly lymph node metastasis, not hematogenous metastasis like liver metastasis $[7,8]$. We report a rare case of T1N0 colon cancer without lymphovascular invasion with synchronous liver metastasis not detected by preoperative imaging study. Written informed consent was obtained.

\section{CASE REPORT}

A 54-year-old man presented to our department for treatment of sigmoid colon cancer following an endoscopic submucosal dissection (ESD). He had no specific personal or family history of malignancy. He received colonoscopy and abdominal computed to- 
mography (CT) scan in December 2013 because of blood tinged stool. Several polyps were found and a 3-cm sized pedunculated mass was found at sigmoid colon on colonoscopy (Fig. 1A). Abdominal CT revealed an intraluminal mass at sigmoid colon, and others were unremarkable (Fig. 1B). ESD and several polypectomies were done. Microscopically, the pedunculated mass from the sigmoid colon revealed a moderately differentiated adenocarcinoma arising from a tubulovillous adenoma. The depth of submucosal invasion was 2,000 $\mu \mathrm{m}$ from the muscularis mucosa and lymphovascular invasion was not identified. The resection margin was not involved (Fig. 2). The other polyps were adenoma with low grade dysplasia. His carcinoembryonic antigen (CEA) level was 1.4 $\mathrm{ng} / \mathrm{mL}$ (normal level: $<1.5 \mathrm{ng} / \mathrm{mL}$ ). He underwent FDG-positron emission tomography/CT (FDG-PET/CT) and there was no evidence of distant metastasis.

We performed a laparoscopic anterior resection with lymph node dissection 3 weeks after ESD. The final pathologic report indicated that there was no residual tumor and none of the 23 lymph nodes were confirmed as metastasis. There was no lymphatic, venous, or perineural invasion and the resection margins were clear (Fig. 3). His pathological stage was T1N0M0, stage I (American Joint Committee on Cancer 8th).

After 3 months, we checked routinely the patient's CEA level and his CEA level was elevated $(7.26 \mathrm{ng} / \mathrm{mL}$, normal level: $<1.5$ $\mathrm{ng} / \mathrm{mL}$ ). Then, he underwent abdominal CT and FDG-PET/CT. The abdominal CT and FDG-PET/CT showed multiple metastases in both hepatic lobes (SUVmax: 5.6) without evidence of local recurrence or lymphadenopathy (Figs. 4, 5). We strongly suspected a synchronous liver metastasis not detected by imaging study as opposed to a systemic recurrence. Surgery was impossible, because the finding was multiple hepatic metastases. Our plan was palliative, targeted-agent chemotherapy. But, unfortunately the patient has elected chemotherapy in another hospital, and the progress since then is unknown.

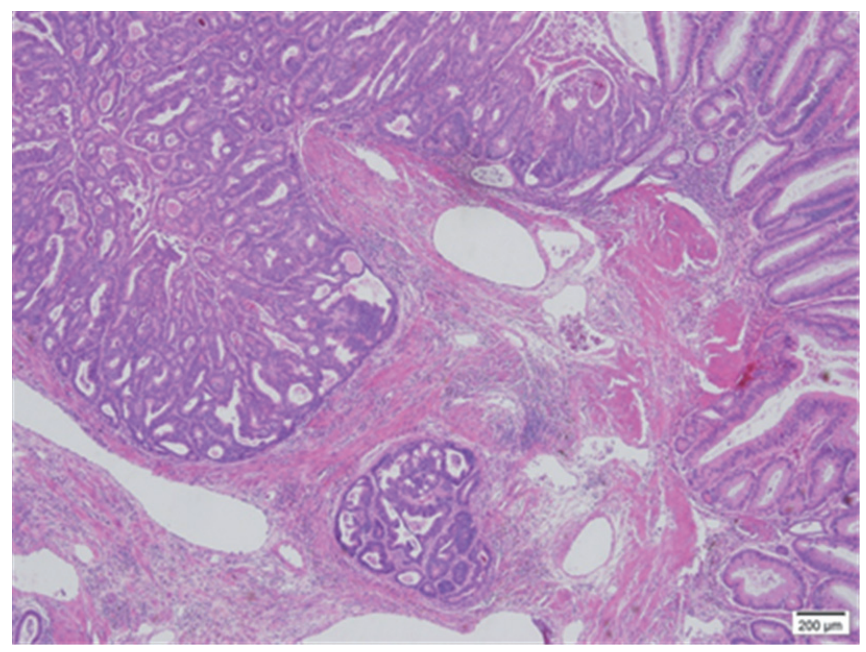

Fig. 2. Histopathologic findings after endoscopic mucosal resection. Moderately differentiated adenocarcinoma was found arising from tubulovillous adenoma with early stromal invasion $\left(H \& E_{1} \times 40\right)$.
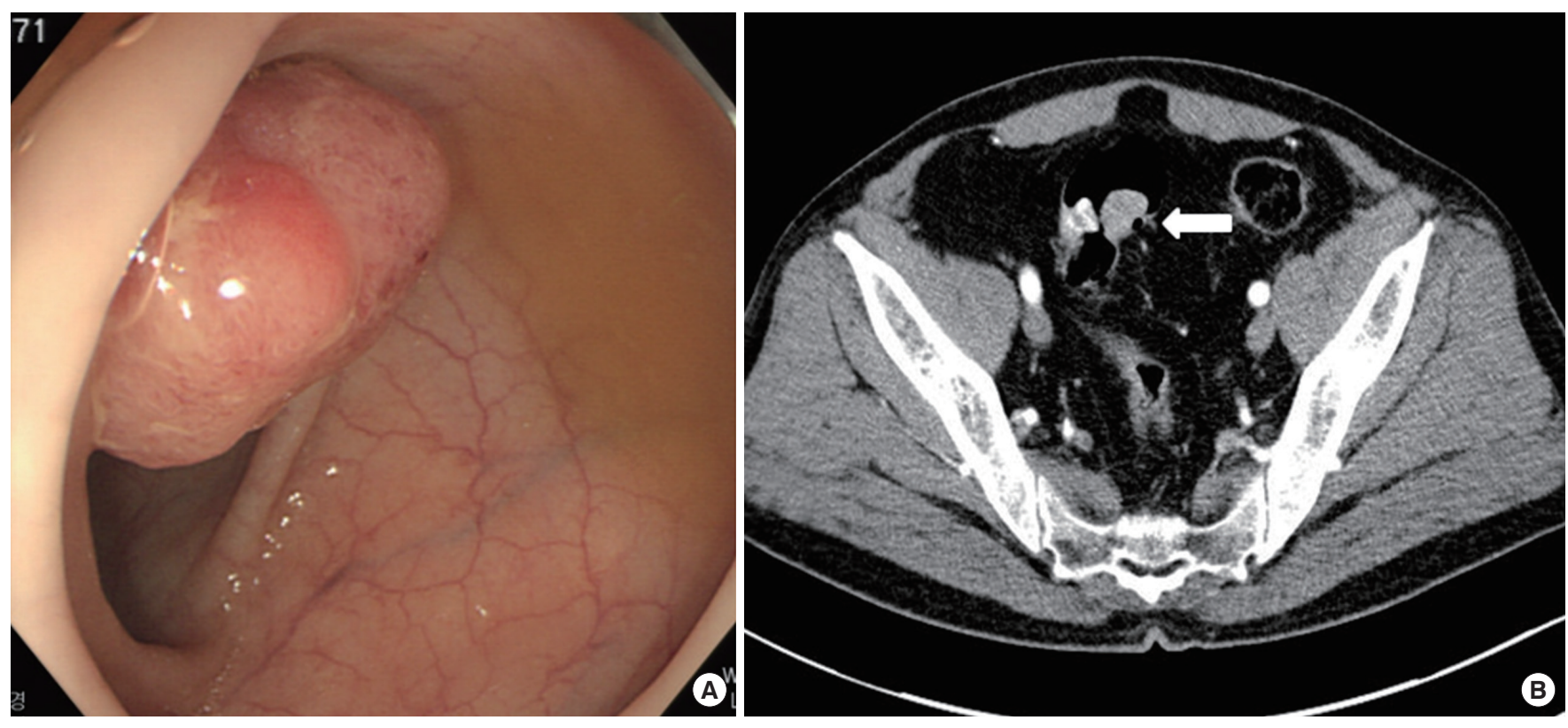

Fig. 1. Initial findings of sigmoid colon mass. (A) A 3-cm sized pedunculated mass was shown in the sigmoid colon from a 30-cm anal verge on colonoscopy. (B) An intraluminal mass (arrow) was found at sigmoid colon on abdominal computed tomography. 

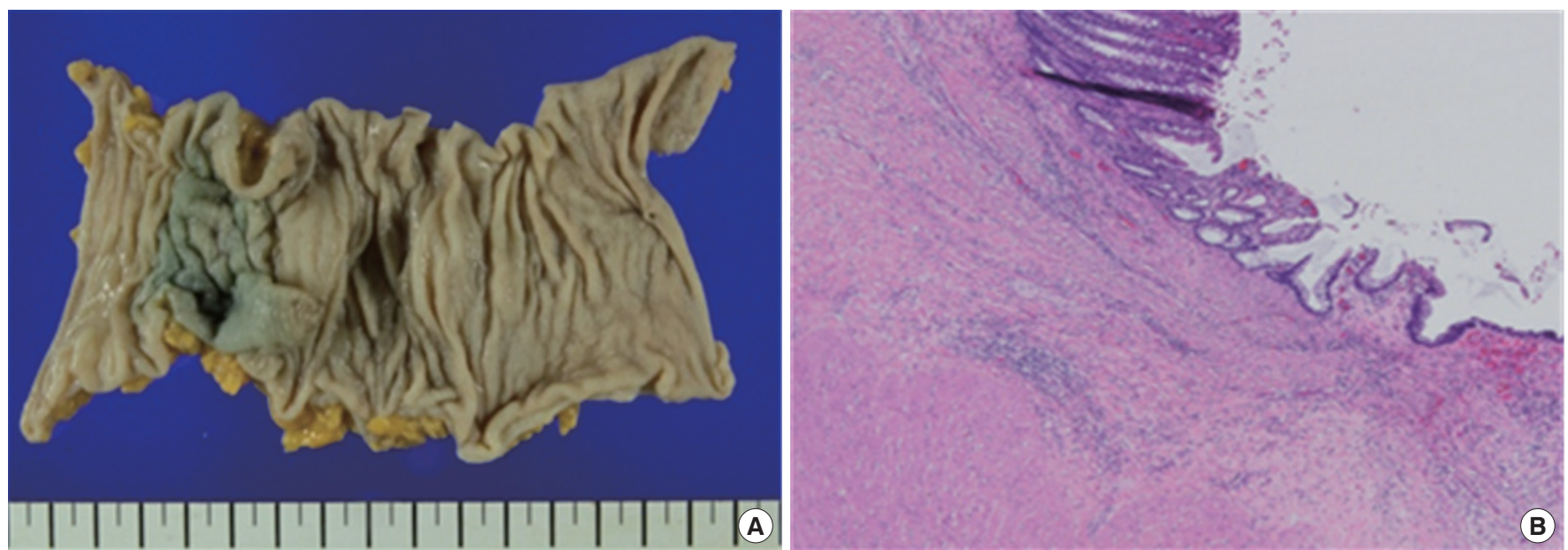

Fig. 3. Surgical specimen of the sigmoid colon. (A) Focal fibrotic scar lesion was shown. (B) Pathologic examination revealed chronic inflammation and granulation tissue $\left(H \& E_{1} \times 40\right)$. No residual tumor was detected.

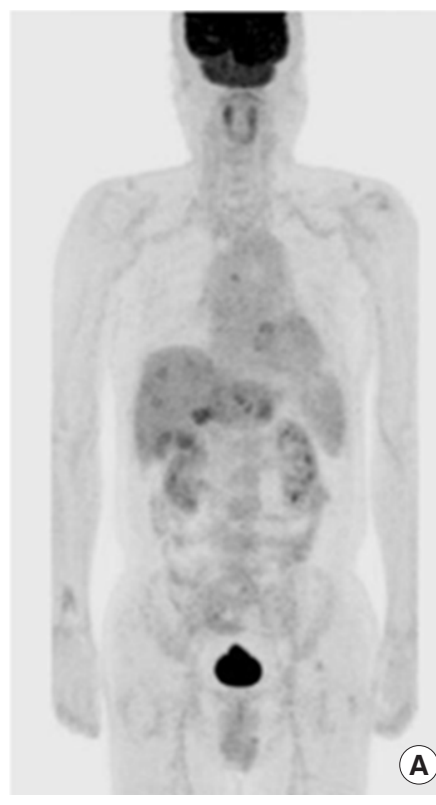

Fig. 4. (A-C) Positron emission tomography-computed tomography (PET/CT) 3 months after radical resection. $\mathrm{PET} / \mathrm{CT}$ revealed multiple newly formed hypermetabolic lesions (arrows) were seen in both hepatic lobes (SUVmax: 5.6). No abnormal FDG uptake was seen in anastomosis site of colon.

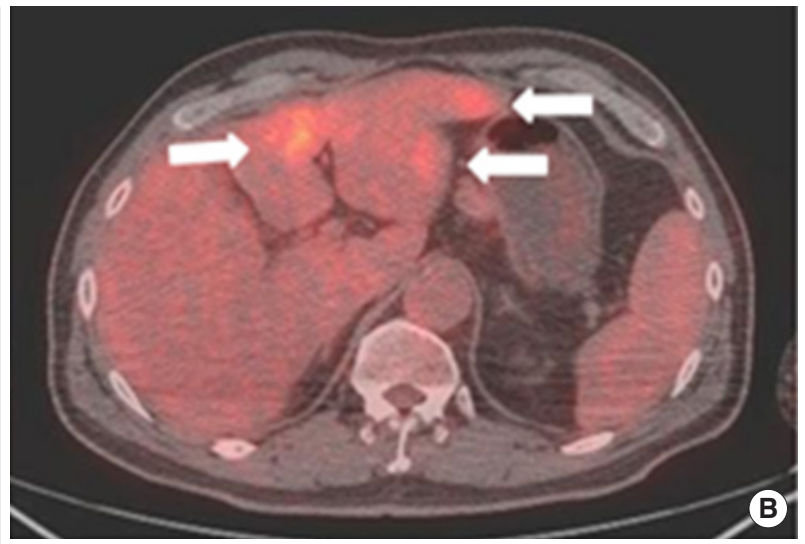

(A)

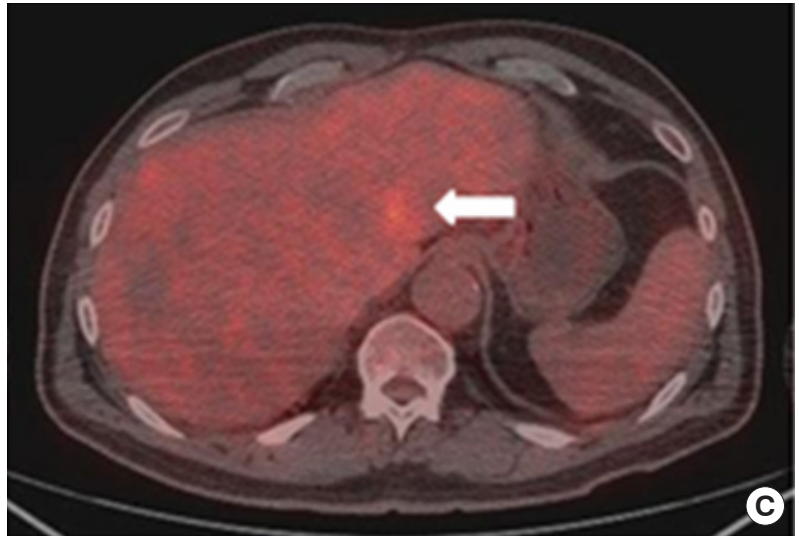

\section{DISCUSSION}

T1 colorectal cancer is considered to have a good prognosis, and the recurrence rate has been reported to be $1.3 \%$ after radical surgery [2]. The form of metastasis in T1 colorectal cancer is mainly lymph node metastasis, but some patients exhibit liver metastasis. A multicenter study in Japan reported that 153 (8.5\%) of 1,806 pa-

tients with $\mathrm{T} 1$ colorectal cancer had lymph node metastases, but $22(1.2 \%)$ of them had liver metastases, and three of them $(0.2 \%)$ had synchronous liver metastases. At the point of synchronous or metachronous distant metastases from T1 colorectal cancer, lymph node metastases, depth of invasion and venous invasion are thought to be the risk factors. Okano et al. [6] reported that seven of 213 patients (3.3\%) with T1 colorectal cancer without lymph 
$\mathrm{KJCO}$
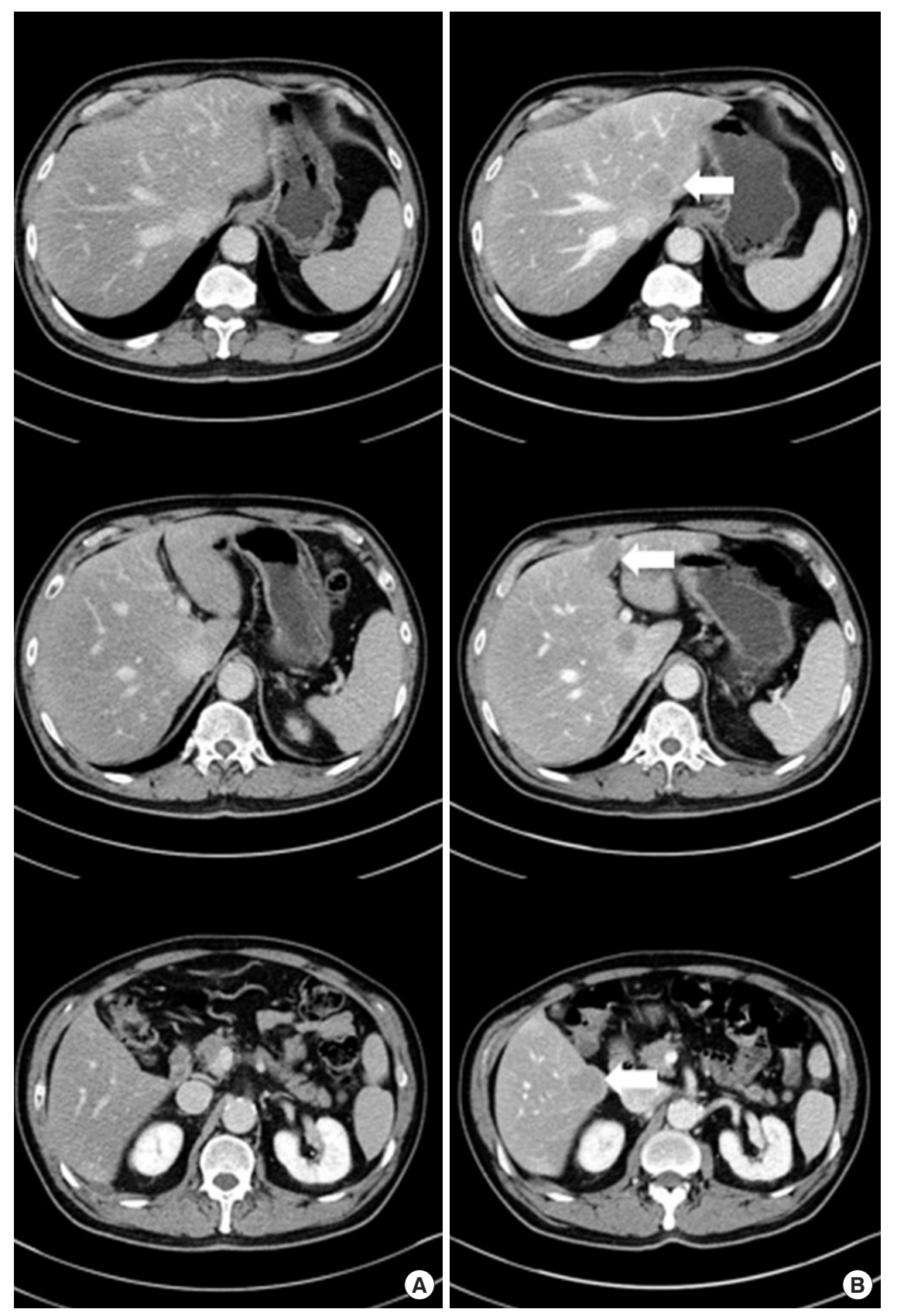

Fig. 5. (A) Preoperative abdomen CT (liver view). (B) Abdomen CT with 3 months after surgery for colon cancer (arrow, multiple hepatic metastases). CT, computed tomography. node metastasis had liver metastases and two $(0.2 \%)$ of them had synchronous liver metastases. Okano et al. [6] reported that venous invasion and the grade of differentiation of the tumor are closely related to liver metastases in $\mathrm{T} 1$ colorectal cancer without lymph node metastasis. In our case, the final pathologic report indicated that the mass was moderately differentiated adenocarcinoma with 2,000 $\mu \mathrm{m}$ depth of submucosal invasion but there was no lymphatic or venous invasion. Submucosal invasion and grade of differentiation were thought as risk factors for liver metastases.

Synchronously detected metastases were defined as metastases detected prior to or during resection of the primary tumor, and in the case of non-resected patients as detected prior to or concurrently with the primary tumor. There were no metastases in the preoperative evaluation. However, less than 6 months later, multiple liver metastases were detected in the abdomen CT, PET/CT. Usually, it is reasonable to assume rapid progressive synchronous liver metastases rather than metachronous liver metastases.

Liver resection is the only potentially curative treatment for colorectal liver metastasis and in selected groups, the 5-year median survival has been reported to be up to $30 \%$ (range, 15\%-67\%) [9]. If left untreated, virtually all patients with liver metastases would be dead within 5 years with a median survival of 8 months [10]. In 
such case, preoperative evaluation of liver metastasis and prediction of metastasis is very important on patient selection to avoid inappropriate treatment. A multi-modality strategy is recommended since no single modality can accurately detect all colorectal liver metastases [11].

In preparing this case report, we looked closely at the preoperative evaluation conducted in collaboration with radiology and nuclear medicine. There was a high possibility of liver metastatic lesions at the time of preoperative evaluation, but the review did not reveal any suspected lesions. Although this is a rare case, many studies are needed to find such rapid synchronous metastases that are not detected in current and routine $\mathrm{CT}$ and PET/CT.

Magnetic resonance imaging (MRI) is particularly useful in characterizing indeterminate lesions detected on other modalities such as CT or unenhanced ultrasonography; it can also identify some lesions not visualized on either of these modalities [12]. Cho et al. [13]. reported that 121 equivocal hepatic lesions were detected on preoperative abdominal CT in 65 out of 494 patients (13.2\%) who were diagnosed with colorectal cancer, and 11 lesions were classified as definitive metastatic lesion based on subsequent liver MRI. A prospective randomized trial [14] reported that 35 patients who were diagnosed with rectal cancer were divided into two groups, abdominal CT group (15 patients) and diffusion weighted liver MRI group (DWMR; 20 patients). The per-patient sensitivity/ specificity was $50 \% / 100 \%$ for CT, and $100 \% / 94 \%$ and $100 \% / 100 \%$ for reader 1 and 2 for DWMR. The per-lesion sensitivity of CT and DWMR were $17 \%$ and $89 \%$, respectively. In a meta-analysis, on a per-patient basis, FDG-PET had significantly higher sensitivity compared with that of the other modalities in the detection of colorectal liver metastasis. Yet, on a per-lesion basis, sensitivity estimates for MRI with contrast agent were significantly superior to those for helical CT with $45 \mathrm{~g}$ of iodine or less [4]. In our case, we did not take liver MRI by conventional preoperative evaluation protocol (National Comprehensive Cancer Network Guideline, 2019) and we could not diagnose liver metastases on preoperative abdominal CT and FDG-PET/CT. We believe that preoperative liver MRI with contrast agent can be useful in selected patients with liver metastases not visualized on abdominal CT.

According to the NCCN guideline 2019, stage 1 colon cancer requires more careful surveillance in stage 1 colon cancer, since CEA or other specific imaging studies are not included in postoperative follow-up guidelines. Besides image study, investigating metastasis associated in colon cancer 1 (MACC1) and MET expression in colorectal adenoma, Tis, early-stage invasive (T1 and T2), and advanced adenocarcinoma with liver metastasis can be done using immunohistochemistry. Stepwise elevation of MACC1 expression in key points of colorectal cancer development suggests that MACC1 may contribute to cancer initiation and early invasive growth. High expression of both MACC1 and MET may relate to distant metastasis [15]. The possibility of metastasis and prognosis of colorectal cancer through MACC1 may be helpful in increasing the survival rate of patients by prompt and appropriate testing. Unfortunately, we did not routinely test MACC1 and MET oncogene, so the above tests could not be performed in these patients. As such, there is regret that more appropriate tests and treatments would have been faster.

In conclusion, there are few well-organized reports about T1 colorectal cancer with synchronous liver metastases, because T1 colorectal cancer with synchronous liver metastasis is very rare. If observations of this disease pattern increase, it will be possible to assess the risk factor of liver metastases in $\mathrm{T} 1$ colorectal cancer, and protocols for preoperative evaluation should be changed to allow more accurate imaging of liver metastases. Even with T1 colorectal cancer, it is possible to predict the prognosis and the possibility of metastasis through genetic studies, and to provide patients with faster and more appropriate treatments if appropriate image studies such as liver MRI are considered.

\section{CONFLICT OF INTEREST}

No potential conflict of interest relevant to this article was reported.

\section{REFERENCES}

1. Jemal A, Bray F, Center MM, Ferlay J, Ward E, Forman D. Global cancer statistics. CA Cancer J Clin 2011;61:69-90.

2. Kobayashi H, Mochizuki H, Sugihara K, Morita T, Kotake K, Teramoto T, et al. Characteristics of recurrence and surveillance tools after curative resection for colorectal cancer: a multicenter study. Surgery 2007;141:67-75.

3. Oxley EM, Ellis H. Prognosis of carcinoma of the large bowel in the presence of liver metastases. Br J Surg 1969;56:149-52.

4. Bipat S, van Leeuwen MS, Comans EF, Pijl ME, Bossuyt PM, Zwinderman AH, et al. Colorectal liver metastases: CT, MR imaging, and PET for diagnosis: meta-analysis. Radiology 2005;237: 123-31.

5. Ruers T, Bleichrodt RP. Treatment of liver metastases, an update on the possibilities and results. Eur J Cancer 2002;38:1023-33.

6. Okano K, Shimoda T, Matsumura Y. Clinicopathologic and immunohistochemical study of early colorectal cancer with liver metastases. J Gastroenterol 1999;34:334-40.

7. Sugimoto K, Kawai M, Takehara K, Tashiro Y, Munakata S, Ishiyama S, et al. T1 colorectal cancer with synchronous liver metastasis. Case Rep Gastroenterol 2013;7:266-71. 
8. Ueno H, Mochizuki H, Hashiguchi Y, Shimazaki H, Aida S, Hase K, et al. Risk factors for an adverse outcome in early invasive colorectal carcinoma. Gastroenterology 2004;127:385-94.

9. Simmonds PC, Primrose JN, Colquitt JL, Garden OJ, Poston GJ, Rees M. Surgical resection of hepatic metastases from colorectal cancer: a systematic review of published studies. Br J Cancer 2006; 94:982-99.

10. Simmonds PC. Palliative chemotherapy for advanced colorectal cancer: systematic review and meta-analysis. Colorectal Cancer Collaborative Group. BMJ 2000;321:531-5.

11. Sica GT, Ji H, Ros PR. CT and MR imaging of hepatic metastases. AJR Am J Roentgenol 2000;174:691-8.

12. Ong KO, Leen E. Radiological staging of colorectal liver metastases.
Surg Oncol 2007;16:7-14.

13. Cho JY, Lee YJ, Han HS, Yoon YS, Kim J, Choi Y, et al. Role of gadoxetic acid-enhanced magnetic resonance imaging in the preoperative evaluation of small hepatic lesions in patients with colorectal cancer. World J Surg 2015;39:1161-6.

14. Achiam MP, Logager VB, Skjoldbye B, Moller JM, Lorenzen T, Rasmussen VL, et al. Preoperative CT versus diffusion weighted magnetic resonance imaging of the liver in patients with rectal cancer; a prospective randomized trial. PeerJ 2016;4:e1532.

15. Ren B, Zakharov V, Yang Q, McMahon L, Yu J, Cao W. MACC1 is related to colorectal cancer initiation and early-stage invasive growth. Am J Clin Pathol 2013;140:701-7. 